\title{
Fundamental Solution for the Plane Problem in Magnetothermoelastic Diffusion Media
}

\author{
R. Kumar*, V. Chawla** \\ Department of Mathematics, Kurukshetra University \\ Kurukshetra-136119, Haryana (India) \\ E-mails: *rajneesh_kuk@ rediffmail.com, ${ }^{* *}$ vijay.chawla@ymail.com
}

Received: 22 May 2012; revised: 3 May 2013; accepted: 17 May 2013; published online: 23 September 2013

\begin{abstract}
The aim of the present paper is to study the fundamental solution in orthotropic magneto- thermoelastic diffusion media. With this objective, firstly the two-dimensional general solution in orthotropic magnetothermoelastic diffusion media is derived. On the basis of thegeneral solution, the fundamental solution for a steady point heat source in an infinite and a semiinfinite orthotropic magnetothermoelastic diffusion material is constructed by four newly introduced harmonic functions. The components of displacement, stress, temperature distribution and mass concentration are expressed in terms of elementary functions. From the present investigation, some special cases of interest are also deduced and compared with the previously obtained results. The resulting quantities are computed numerically for infinite and semi-infinite magnetothermoelastic material and presented graphically to depict the magnetic effect.
\end{abstract}

Key words: fundamental solution, orthotropic, magnetothermoelastic diffusion, semi-infinite, infinite

\section{INTRODUCTION}

Fundamental solutions or Green's functions play an important role in the solution of numerous problems in the mechanics and physics of solids. They are a basic building block of many further works. For example, fundamental solutions can be used to construct many analytical solutions of practical problems when boundary conditions are imposed. They are essential in the boundary element method as well as the study of cracks, defects and inclusions.

Ding, Chen and Liang [1] derived the general solutions for coupled equations in piezoelectric media. Dunn and Wienecke [2] derived the half space Green's functions for transversely isotropic piezoelectric solid. Pan and Tanon [3] studied the Green's functions for a threedimensional problem in general anisotropic piezoelectric solids.

When thermal effects are considered, Ding, Guo and Hou [4] obtained a three-dimensional general solution in transversely isotropic piezothermoelastic media. Sharma [5] investigated the fundamental solution for trans- versely isotropic thermoelastic material in an integral form. Chen et. al. [6] derived the three dimensional general solution for transversely isotropic thermoelastic materials. Hou et. al. [7, 8] investigated the Green's function for two and three-dimensional problems for a steady Point heat source in the interior of a semi-infinite thermoelastic materials. Also, Hou et. al. [9] investigated the two dimensional general solution and fundamental solution for orthotropic thermoelastic materials.

The theory of magnetothermoelasticity is concerned with the interacting effects of the applied magnetic field on the elastic and thermoelastic deformation of a solid body. This theory has drawn the attention of many researchers because of its extensive uses in diverse fields, such as geophysics for understanding the effect of Earth's magnetic field on seismic waves, damping of acoustic waves in a magnetic field. Kolaski and Nowacki [10] studied the magnetothermoelastic disturbance in a perfectly conducting elastic half-space in contact with vacuum due to applied thermal disturbance on the plane boundary. Othman and Song [11] investigated the reflection of magnetothermoelastic waves with two relaxation 
times. Hou et. al. [12] investigated the general solution and fundamental solution for orthotropic magnetothermoelastic materials.

Diffusion is the spontaneous movement of matter (particles) from a region of high concentration to low concentration. Diffusion occurs in response to a concentration gradient expressed as the change in concentration due to the change in position. The example of diffusion is heat transport or movement transport. The net flux of transported quantity (atoms, energy or electrons) is equal to a physical property (diffusivity, thermal conductivity and electric conductivity) multiplied by a gradient (concentration, thermal and electric field gradient). The concept of thermodiffusion is used to describe the process of thermo-mechanical treatment of metals. The study of this phenomenon is of great concern due to its many geophysical and industrial applications. For example, oil companies are interested in the process of thermodiffusion for more efficient extraction of oils from oils deposits. The thermodiffusion process also helps the investigation in the field associated with the advent of semiconductor devices and that advancement of microelectronics. Most of the research associated with the presence of concentration and temperature gradients have been made with metals and alloys.

Nowacki [13-16] developed the theory of thermoelastic diffusion by using the coupled thermoelastic model. Sherief et. al. [17] developed the generalized theory of thermoelastic diffusion with one relaxation time which allows finite speeds of propagation of waves. Kumar and Kansal [18] derived the basic equations for generalized thermoelastic diffusion. When diffusion effects are considered, Kumar and Chawla [19] discussed the Plane wave propagation in the anisotropic three-phase-lag model. Kumar and Chawla [20] investigated the fundamental solution in orthotropic thermoelastic diffusion material. Kumar and Chawla [21] studied the Green's function for a two-dimensional problem in orthotropic thermoelastic diffusion material. However, the important fundamental solution for a two-dimensional problem in magnetothermoelastic diffusion material has not been discussed so far.

The fundamental solution for two-dimensional problem in orthotropic magnetothermoelastic diffusion medium is investigated in this paper. Based on the two-dimensional general solution of orthotropic magnetothermoelastic diffusion media, the fundamental solutions for a steady point heat source acting in an infinite plane and on the surface of a semi-infinite plane are obtained by four newly introduced harmonic functions. From the present investigation, some special cases of interest are also deduced.

\section{BASIC EQUATIONS}

Following Nayfeh and Nasser [22], Maxwell equations in vector form can be written as

$$
\begin{gathered}
\nabla \times \vec{H}=\vec{J}+\dot{\vec{D}}, \\
\nabla \times \vec{E}=-\dot{\vec{B}}, \\
\nabla \cdot \vec{B}=0 \\
\nabla \cdot \vec{D}=\rho_{e} \\
\vec{B}=\mu_{e} \vec{H}, \quad \vec{H}=\vec{H}_{0}+\vec{h} \\
\vec{D}=\varepsilon \vec{E}
\end{gathered}
$$

and the modified Ohm's law

$$
\vec{J}=\sigma[\vec{E}+\dot{\vec{u}} \times \vec{B}]
$$

where $\nabla$ is the del operator, $\vec{H}$ is the magnetic intensity vecor; $\vec{J}$ is the conduction current density, $\vec{D}$ and $\vec{E}$ are, respectively, the electric flux and electric density vecor $(\dot{\vec{D}}$ is commonly termed the electric displacement current), $\vec{B}$ is the magnetic field density; $\rho_{e}$ is the charge density, the constants $\mu_{e}, \varepsilon$ and $\sigma$ which characterize the medium are known as the magnetic permeability, electric permittivity and electric conductivity, respectively, $\vec{u}$ is the displacement vector.

Using equation (5), equation (7) can be linearized by neglecting the small quantities of the second order giving

$$
\vec{J}=\sigma\left[\vec{E}+\mu_{e} \dot{\vec{u}} \times \vec{H}_{0}\right]
$$

The above equations (1)-(8) are supplemented by equations of motion and constitutive relations (in case of perfectly electroconducting i.e. $\sigma \rightarrow \infty$ ) in the theory of generalized thermoelastic diffusion, taking into account the Lorentz force (Eringen [23]),

(i) Constitutive relations

$$
\sigma_{i j}=c_{i j k m} e_{k m}+a_{i j} T+b_{i j} C,
$$

(ii) Equations of motion

$$
c_{i j k m} e_{k m, j}+a_{i j} T_{, j}+b_{i j} C_{, j}+\breve{F}_{i}=\rho \ddot{u}_{i},
$$

(iii) Equation of heat conduction

$$
\rho C_{E} \dot{T}+a T_{0} \dot{C}-a_{i j} T_{0} \dot{e}_{i j}=K_{i j} T_{, i j},
$$

(iv) Equation of mass diffusion

$$
-\alpha_{i j}^{*} b_{k m} e_{k m, i j}-\alpha_{i j}^{*} b C,_{i j}+\alpha_{i j}^{*} a T,{ }_{i j}=-\dot{C} .
$$

Here, $F_{i}=(\vec{J} \times \vec{B})_{i}, c_{i j k m}\left(=c_{k m i j}=c_{j i k m}=c_{i j m k}\right)$ are elastic parameters, $a_{i j}\left(=a_{j i}\right), b_{i j}\left(=b_{j i}\right)$ are, respectively, the tensor of thermal and diffusion moduli. $\rho$ is the density and $C_{E}$ is the specific heat at constant strain, $a$ and $b$ are, respectively, coefficient describing the measure of thermoelastic 
diffusion effects and of diffusion effects, $T_{0}$ is the reference temperature assumed to be such that $\left|\frac{T}{T_{0}}\right|<<1, K_{i j}\left(=K_{j i}\right)$, $\sigma_{i j}\left(=\sigma_{j i}\right)$ and $e_{i j}=\frac{u_{i, j}+u_{j, i}}{2}$ denote the components of thermal conductivity, stress and strain tensor, respectively, $T(x, y, z, t)$ is the temperature change from the reference temperature $T_{0}$ and $C$ is the mass concentration. $u_{i}$ are components of displacement vector, $\alpha_{i j}^{*}\left(=\alpha_{j i}^{*}\right)$ are diffusion parameters. $\breve{F}_{i}$ are components of Lorentz force.

In the above equations symbol (",") followed by a suffix denotes differentiation with respect to spatial coordinate and a superposed dot (“.”) denotes the derivative with respect to time, respectively.

\section{FORMULATION OF THE PROBLEM}

We consider a homogenous orthotropic magnetothermoelastic diffusion medium. Let us take $O x y z$ as the frame of reference in Cartesian coordinates, the origin $O$ being any point on the plane boundary.

For a two-dimensional problem, we assume the displacement vector, temperature distribution, mass concentration, applied magnetic field $\vec{H}_{0}$, induced magnetic field $\vec{h}$, current electric density vector $\vec{E}$ and $\vec{J}$ conduction current density vector are respectively of the form

$$
\begin{gathered}
\vec{u}=(u, 0, w), \quad T(x, z, t), \quad C(x, z, t) \\
\vec{H}_{0}=\left(0, H_{0}, 0\right), \quad \vec{h}=(0, h, 0), \\
\vec{E}=\left(E_{1}, 0, E_{3}\right), \quad \vec{J}=\left(J_{1}, 0, J_{3}\right) .
\end{gathered}
$$

It can be easily seen from equation (13) that Lorentz force will have non-vanishing components in $x-z$ direction, that is

$$
\begin{gathered}
\breve{F}_{1}=\mu_{0} H_{0}^{2}\left(\frac{\partial e}{\partial x}-\varepsilon_{0} \mu_{0} \frac{\partial^{2} u}{\partial t^{2}}\right), \\
\breve{F}_{3}=\mu_{0} H_{0}^{2}\left(\frac{\partial e}{\partial z}-\varepsilon_{0} \mu_{0} \frac{\partial^{2} w}{\partial t^{2}}\right), \\
e=\frac{\partial u}{\partial x}+\frac{\partial w}{\partial z} .
\end{gathered}
$$

Moreover, we are discussing steady problem

$$
\frac{\partial u}{\partial t}=\frac{\partial w}{\partial t}=\frac{\partial C}{\partial t}=\frac{\partial T}{\partial t}=0 .
$$

We define the dimensionless quantities as:

$$
\begin{gathered}
\left(x^{\prime}, z^{\prime}, u^{\prime} w^{\prime}\right)=\frac{\omega_{1}^{*}}{v_{1}}(x, z, u, w), \\
\left(T^{\prime}, C^{\prime}\right)=\frac{1}{c_{11}}\left(a_{1} T, b_{1} C\right),
\end{gathered}
$$

$$
\sigma_{i j}^{\prime}=\frac{\sigma_{i j}}{a_{1} T_{0}}, \quad H^{\prime}=\frac{a_{1} v_{1}}{c_{11} K_{1} \omega_{1}^{*}} H
$$

where

$$
v_{1}^{2}=b_{1}, \quad \omega_{1}^{*}=\frac{a c_{11}}{K_{1}} .
$$

Equations (9) - (12) for orthotropic magnetothermoelastic diffusion material, with the aid of (13)-(16), after suppressing the primes, yields

$$
\begin{gathered}
\left(\delta_{1} \frac{\partial^{2}}{\partial x^{2}}+\delta_{2} \frac{\partial^{2}}{\partial z^{2}}\right) u+\left(\delta_{3} \frac{\partial^{2}}{\partial x \partial z}\right) w \\
-\left(\frac{\partial}{\partial x}\right) C-\left(\frac{\partial}{\partial x}\right) T=0 \\
\left(\delta_{3} \frac{\partial^{2}}{\partial x \partial z}\right) u+\left(\delta_{2} \frac{\partial^{2}}{\partial x^{2}}+\delta_{4} \frac{\partial^{2}}{\partial z^{2}}\right) w \\
-\varepsilon_{1}\left(\frac{\partial}{\partial z}\right) C-\varepsilon_{2}\left(\frac{\partial}{\partial z}\right) T=0 \\
\left(\frac{\partial^{2}}{\partial x^{2}}\right) T+\varepsilon_{3}\left(\frac{\partial^{2}}{\partial z^{2}}\right) T=0 \\
\frac{\partial}{\partial x}\left(q_{1}^{*} \frac{\partial^{2}}{\partial x^{2}}+q_{3}^{*} \frac{\partial^{2}}{\partial z^{2}}\right) u+\frac{\partial}{\partial z}\left(q_{2}^{*} \frac{\partial^{2}}{\partial x^{2}}+q_{4}^{*} \frac{\partial^{2}}{\partial z^{2}}\right) w \\
-\left(q_{5}^{*} \frac{\partial^{2}}{\partial x^{2}}+q_{6}^{*} \frac{\partial^{2}}{\partial z^{2}}\right) C+\left(q_{7}^{*} \frac{\partial^{2}}{\partial x^{2}}+q_{8}^{*} \frac{\partial^{2}}{\partial z^{2}}\right) T=0
\end{gathered}
$$

where

$$
\begin{aligned}
& \delta_{1}=1+\frac{\mu_{0} H_{0}^{2}}{c_{11}}, \\
& \left(\delta_{2}, \delta_{3}, \delta_{4}\right)=\frac{1}{c_{11}}\left(c_{55}, c_{13}+c_{55}\right. \\
& \left.+\mu_{0} H_{0}^{2} \omega_{1}^{*}, c_{33}+\mu_{0} H_{0}^{2} \omega_{1}^{*}\right), \\
& \varepsilon_{1}=\frac{b_{3}}{b_{1}}, \quad \varepsilon_{2}=\frac{a_{3}}{a_{1}}, \quad \varepsilon_{3}=\frac{K_{3}}{K_{1}}, \\
& \left(q_{1}^{*}, q_{2}^{*}\right)=\frac{\alpha_{1}^{*} \omega_{1}^{*}}{c_{11}}\left(b_{1}, b_{3}\right), \\
& \left(q_{3}^{*} q_{4}^{*}\right)=\frac{\alpha_{3}^{*} \omega_{1}^{*}}{c_{11}}\left(b_{1}, b_{3}\right), \\
& \left(q_{5}^{*}, q_{6}^{*}\right)=\frac{\omega_{1}^{*} b}{b_{1}}\left(\alpha_{1}^{*}, \alpha_{3}^{*}\right),\left(q_{7}^{*}, q_{8}^{*}\right)=\frac{a \omega_{1}^{*}}{a_{1}}\left(\alpha_{1}^{*}, \alpha_{3}^{*}\right) .
\end{aligned}
$$

The equations (17)-(20) can be written as

$$
D\{u, w, C, T\}^{t r}=0,
$$


where $D$ is the differential operator matrix given by

$$
\left[\begin{array}{cccc}
\delta_{1} \frac{\partial^{2}}{\partial x^{2}}+\delta_{2} \frac{\partial^{2}}{\partial z^{2}} & \delta_{3} \frac{\partial^{2}}{\partial x \partial z} & -\frac{\partial}{\partial x} & -\frac{\partial}{\partial x} \\
\delta_{3} \frac{\partial^{2}}{\partial x \partial z} & \delta_{2} \frac{\partial^{2}}{\partial x^{2}}+\delta_{4} \frac{\partial^{2}}{\partial z^{2}} & -\varepsilon_{1} \frac{\partial}{\partial z} & -\varepsilon_{2} \frac{\partial}{\partial z} \\
\frac{\partial}{\partial x}\left(q_{1}^{*} \frac{\partial^{2}}{\partial x^{2}}+q_{3}^{*} \frac{\partial^{2}}{\partial z^{2}}\right) & \frac{\partial}{\partial z}\left(q_{2}^{*} \frac{\partial^{2}}{\partial x^{2}}+q_{4}^{*} \frac{\partial^{2}}{\partial z^{2}}\right) & -\left(q_{5}^{*} \frac{\partial^{2}}{\partial x^{2}}+q_{6}^{*} \frac{\partial^{2}}{\partial x^{2}}\right) & \left(q_{7}^{*} \frac{\partial^{2}}{\partial x^{2}}+q_{8}^{*} \frac{\partial^{2}}{\partial x^{2}}\right) \\
0 & 0 & 0 & \left(\frac{\partial^{2}}{\partial x^{2}}+\varepsilon_{3} \frac{\partial^{2}}{\partial z^{2}}\right)
\end{array}\right] .
$$

Equation (21) is a homogeneous set of differential equations in $u, w, C, T$. The general solution by the operator theory as follows

$$
\begin{aligned}
u=A_{i 1} F, w= & A_{i 2} F, C=A_{i 3} F, T=A_{i 4} F, \\
& (i=1,2,3,4)
\end{aligned}
$$

where $A_{i j}$ are algebraic cofactors of the matrix $D$, of which the determinant is

$$
\begin{gathered}
|D|=\left(a^{*} \frac{\partial^{6}}{\partial z^{6}}+b^{*} \frac{\partial^{6}}{\partial x^{2} \partial z^{4}}\right. \\
\left.+c^{*} \frac{\partial^{6}}{\partial x^{4} \partial z^{2}}+d^{*} \frac{\partial^{6}}{\partial x^{6}}\right) \times\left(\frac{\partial^{2}}{\partial x^{2}}+\varepsilon_{3} \frac{\partial^{2}}{\partial z^{2}}\right),
\end{gathered}
$$

where

$$
\begin{aligned}
& a^{*}=\delta_{2}\left(\varepsilon_{1} q_{4}^{*}-\delta_{4} q_{6}^{*}\right), \\
& b^{*}=\delta_{1}\left(\varepsilon_{1} q_{4}^{*}-\delta_{4} q_{6}^{*}\right)-\delta_{2}\left(\delta_{2} q_{6}^{*}+\delta_{4} q_{5}^{*}\right) \\
& +\delta_{2}\left(\varepsilon_{1} q_{2}^{*}+\delta_{3} q_{6}^{*}\right)-q_{7}^{*}\left(\delta_{4}+\delta_{2} \varepsilon_{1}\right)+\delta_{3} q_{4}^{*}, \\
& c^{*}=\delta_{1}\left(\varepsilon_{1} q_{2}^{*}-\delta_{4} q_{5}^{*}\right)-\delta_{2}\left(\delta_{1} q_{6}^{*}+\delta_{2} q_{5}^{*}\right) \\
& +\delta_{2}\left(\delta_{3} q_{5}^{*}-\varepsilon_{1} q_{1}^{*}\right)+, \delta_{3} q_{2}^{*}-\delta_{4} q_{1}^{*}-\delta_{2} q_{7}^{*}, \\
& d=-\delta_{2}\left(\delta_{1} q_{5}^{*}+q_{1}^{*}\right) .
\end{aligned}
$$

The function $F$ in equation (23) satisfies the following homogeneous equation

$$
|D| F=0 .
$$

It can be seen that if $i=1,2,3$ are taken in equation (23), three general solutions are obtained in which $T=0$. These solutions are identical to those without thermal fact and are not discussed here. Therefore if $i=4$ should be taken in equation (23), the following solution is obtained

$$
\begin{gathered}
u=\left(p_{1} \frac{\partial^{4}}{\partial x^{4}}+q_{1} \frac{\partial^{4}}{\partial z^{2} \partial x^{2}}+r_{1} \frac{\partial^{4}}{\partial z^{4}}\right) \frac{\partial F}{\partial x}, \\
w=\left(p_{2} \frac{\partial^{4}}{\partial x^{4}}+q_{2} \frac{\partial^{4}}{\partial z^{2} \partial x^{2}}+r_{2} \frac{\partial^{4}}{\partial z^{4}}\right) \frac{\partial F}{\partial z}, \\
C=\left(p_{3} \frac{\partial^{6}}{\partial z^{6}}+q_{3} \frac{\partial^{6}}{\partial z^{4} \partial x^{2}}+r_{3} \frac{\partial^{6}}{\partial z^{2} \partial x^{4}}+l_{3} \frac{\partial^{6}}{\partial x^{6}}\right) F, \\
T=\left(a^{*} \frac{\partial^{6}}{\partial z^{6}}+b^{*} \frac{\partial^{6}}{\partial z^{4} \partial x^{2}}+c^{*} \frac{\partial^{6}}{\partial z^{2} \partial x^{4}}+d^{*} \frac{\partial^{6}}{\partial x^{6}}\right) F,
\end{gathered}
$$

$$
\begin{aligned}
& p_{1}=\left(q_{7}^{*}-q_{5}^{*}\right) \delta_{2}, q_{1}=-\delta_{2}\left(\varepsilon_{1} q_{7}^{*}+q_{5}^{*} \varepsilon_{2}\right) \\
& +\delta_{2}\left(q_{6}^{*}+q_{8}^{*}\right)+\delta_{4}\left(q_{7}^{*}+q_{8}^{*}\right) q_{8}^{*}-\varepsilon_{1} q_{2}^{*}, \\
& r_{1}=-\delta_{2}\left(\varepsilon_{1} q_{8}^{*}+\varepsilon_{2} q_{6}^{*}\right)+\delta_{2} q_{8}^{*}+\delta_{4}\left(q_{6}^{*}+q_{8}^{*}\right) \\
& +\left(q_{4}^{*}+q_{6}^{*}\right) \delta_{4}+\varepsilon_{1} q_{4}^{*}, \\
& p_{2}=\delta_{3}\left(q_{5}^{*}+q_{7}^{*}\right)+q_{1}^{*}\left(\varepsilon_{2}-\varepsilon_{1}\right)-\delta_{1}\left(\varepsilon_{1} q_{7}^{*}+\varepsilon_{2} q_{5}^{*}\right), \\
& r_{2}=-\delta_{1}\left(\varepsilon_{1} q_{8}^{*}+\varepsilon_{2} q_{6}^{*}\right), \\
& q_{2}=-\delta_{1}\left(\varepsilon_{1} q_{8}^{*}+q_{6}^{*} \varepsilon_{2}\right)-\delta_{2}\left(\varepsilon_{1} q_{7}^{*}+\varepsilon_{2} q_{5}^{*}\right) \\
& +\delta_{3}\left(q_{8}^{*}+q_{6}^{*}\right)+q_{7}^{*}\left(\varepsilon_{2}-\varepsilon_{1}\right), \\
& p_{3}=\left(\varepsilon_{2} q_{4}^{*}+\delta_{4} q_{8}^{*}\right) \delta_{2}, \\
& q_{3}=\delta_{1}\left(\delta_{4} q_{8}^{*}+\varepsilon_{2} q_{4}^{*}\right)+\delta_{2}\left(\delta_{2} q_{8}^{*}+\delta_{4} q_{7}^{*}\right) \\
& +\delta_{2}\left(\varepsilon_{2} q_{2}^{*}-\delta_{3} q_{8}^{*}\right)-q_{7}^{*}\left(\delta_{2} \varepsilon_{2}+\delta_{4}\right)+\delta_{3} q_{4}^{*}, \\
& r_{3}=\delta_{1}\left(\delta_{2} q_{8}^{*}+\delta_{4} q_{7}^{*}\right)+\delta_{2}\left(\delta_{2} q_{7}^{*}-\delta_{3} q_{7}^{*}\right) \\
& +q_{2}^{*}\left(\varepsilon_{2} \delta_{1}+\delta_{3}\right)-\delta_{2}\left(q_{1}^{*} \varepsilon_{2}+q_{7}^{*}\right)-\delta_{4} q_{1}^{*}, \\
& l_{3}=\delta_{2}\left(\delta_{1} q_{7}^{*}-q_{1}^{*}\right) .
\end{aligned}
$$

Equation (25) can be rewritten as

$$
\prod_{j=1}^{4}\left(\frac{\partial^{2}}{\partial x^{2}}+\frac{\partial^{2}}{\partial z_{j}^{2}}\right) F=0
$$

where $z_{j}=s_{j} z, s_{4}=\sqrt{\frac{K_{1}}{K_{3}}}$ and $s_{j}(j=1,2,3)$ are three roots (with positive real part) of the following algebraic equation

$$
a^{*} s^{6}-b^{*} s^{4}+c^{*} s^{2}-d^{*}=0 .
$$

As known from the generalized Almansi (Proved by Ding et. al. [1]) theorem, the function $F$ can be expressed in terms of four harmonic functions

$$
\begin{gathered}
F=F_{1}+F_{2}+F_{3}+F_{4} \\
\quad \text { for distinct } s_{j}(j=1,2,3,4) . \\
F=F_{1}+F_{2}+F_{3}+z F_{4} \\
\quad \text { for } s_{1} \neq s_{2} \neq s_{3}=s_{4} . \\
F=F_{1}+F_{2}+z F_{3}+z^{2} F_{4} \\
\quad \text { for } s_{1} \neq s_{2}=s_{3}=s_{4} .
\end{gathered}
$$




$$
\begin{gathered}
F=F_{1}+z F_{2}+z^{2} F_{3}+z^{3} F_{4} \\
\text { for } s_{1}=s_{2}=s_{3}=s_{4} .
\end{gathered}
$$

where $F_{j}(j=1,2,3,4)$ satisfies the following harmonic equations

$$
\left(\frac{\partial^{2}}{\partial x^{2}}+\frac{\partial^{2}}{\partial z_{j}^{2}}\right) F_{j}=0, \quad(j=1,2,3,4) .
$$

The general solution for the case of distinct roots can be derived as follows

$$
\begin{gathered}
u=\sum_{j=1}^{4} p_{1 j} \frac{\partial^{5} F_{j}}{\partial x \partial z_{j}^{4}} \quad w=\sum_{j=1}^{4} s_{j} p_{2 j} \frac{\partial^{5} F_{j}}{\partial z_{j}^{5}} \\
C=\sum_{j=1}^{4} p_{3 j} \frac{\partial^{6} F_{j}}{\partial z_{j}^{6}} \quad T=p_{44} \frac{\partial^{6} F_{4}}{\partial z_{4}^{6}} .
\end{gathered}
$$

The general solution for the other three cases can be derived in the similar way. Equation (31) can be further simplified by taking

$$
p_{1 j} \frac{\partial^{4} F_{j}}{\partial z_{j}^{4}}=\psi_{j} .
$$

Making use of equation (32) in equation (31), we obtain

$$
\begin{gathered}
u=\sum_{j=1}^{4} \frac{\partial \psi_{j}}{\partial x}, \quad w=\sum_{j=1}^{4} s_{j} P_{1 j} \frac{\partial \psi_{j}}{\partial z_{j}} \\
C=\sum_{j=1}^{4} P_{2 j} \frac{\partial^{2} \psi_{j}}{\partial z_{j}^{2}}, \quad T=P_{34} \frac{\partial^{2} \psi_{4}}{\partial z_{4}^{2}}
\end{gathered}
$$

where

$$
P_{1 j}=p_{2 j} / p_{1 j}, P_{2 j}=p_{3 j} / p_{1 j}, P_{34}=p_{44} / p_{14} \text {. }
$$

The function $\psi_{j}$ satisfies the harmonic equations

$$
\left(\frac{\partial^{2}}{\partial x^{2}}+\frac{\partial^{2}}{\partial z_{j}^{2}}\right) \psi_{j}=0, \quad j=1,2,3,4 .
$$

Making use of equations (13), (15) and (16) in equation (9) and after suppressing the primes, with the aid of equation (33), we obtain

$$
\begin{gathered}
\sigma_{x x}=\sum_{j=1}^{4}\left(-f_{1}+f_{1} s_{j}^{2} P_{1 j}\right. \\
\left.-f_{1} P_{3 j}-f_{1} P_{2 j}\right) \frac{\partial^{2} \psi_{j}}{\partial z_{j}^{2}}, \\
\sigma_{z z}=\sum_{j=1}^{4}\left(-f_{2}+h_{1} s_{j}^{2} P_{1 j}\right. \\
\left.-h_{2} P_{3 j}-h_{3} P_{2 j}\right) \frac{\partial^{2} \psi_{j}}{\partial z_{j}^{2}},
\end{gathered}
$$

$$
\sigma_{z x}=\sum_{j=1}^{4} h_{4}\left(1+P_{1 j}\right) s_{j} \frac{\partial^{2} \psi_{j}}{\partial x \partial z_{j}}
$$

where

$$
P_{31}=P_{32}=P_{33}=0
$$

and

$$
\begin{aligned}
& \left(f_{1}, f_{2}, h_{1}, h_{2}, h_{3}, h_{4}\right)= \\
= & \frac{1}{a_{1} T_{0}}\left(c_{11}, c_{13}, c_{33}, \frac{a_{3} c_{11}}{a_{1}}, \frac{b_{3} c_{11}}{b_{1}}, c_{55}\right) .
\end{aligned}
$$

Substituting the values of $\sigma_{x x}, \sigma_{z z}$ and $\sigma_{z x}$ from equation (36) in equations (10)-(11), with the aid of (13), (15) and (16) gives

$$
\begin{gathered}
f_{1}-f_{2} s_{j}^{2} P_{1 j}+f_{1} P_{3 j}+f_{1} P_{2 j}=h_{4}\left(1+P_{1 j}\right) s_{j}^{2}, \\
-f_{2}+h_{1} s_{j}^{2} P_{1 j}-h_{2} P_{3 j}-h_{3} P_{2 j}=h_{4}\left(1+P_{1 j}\right), \\
\left(1-\varepsilon_{3} s_{j}^{2}\right) P_{3 j}=0 .
\end{gathered}
$$

The general solution (36) with the help of (38) can be simplified as

$$
\begin{gathered}
\sigma_{x x}=-\sum_{j=1}^{4} s_{j}^{2} w_{1 j} \frac{\partial^{2} \psi_{j}}{\partial z_{j}^{2}}, \quad \sigma_{z z}=\sum_{j=1}^{4} w_{1 j} \frac{\partial^{2} \psi_{j}}{\partial z_{j}^{2}} \\
\sigma_{z x}=\sum_{j=1}^{4} s_{j} w_{1 j} \frac{\partial^{2} \psi_{j}}{\partial x \partial z_{j}}
\end{gathered}
$$

where

$$
\begin{gathered}
w_{1 j}=\frac{f_{1}-f_{2} s_{j}^{2} P_{1 j}+f_{1} P_{3 j}+f_{1} P_{2 j}}{s_{j}^{2}}= \\
=h_{4}\left(1+\bar{P}_{1 j}\right)=-f_{2}+h_{1} s_{j}^{2} P_{1 j}-h_{2} P_{3 j}-h_{3} P_{3 j} .
\end{gathered}
$$

\section{FUNDAMENTAL SOLUTION FOR A POINT HEAT SOURCE IN AN INFINITE ORTHOTROPIC MAGNETOTHERMOELASTIC DIFFUSION MATERIAL}

As shown in Fig. 1, we consider an infinite orthotropic magnetothermoelastic diffusion material $z \geq 0$. A point heat source $\mathrm{H}$ is applied at the origin and the surface $z=0$ is free, impermeable and thermally insulated. The complete geometry of the problem is shown in Fig. 1. The general solution given by equations (33) and (39) is derived in this section. 


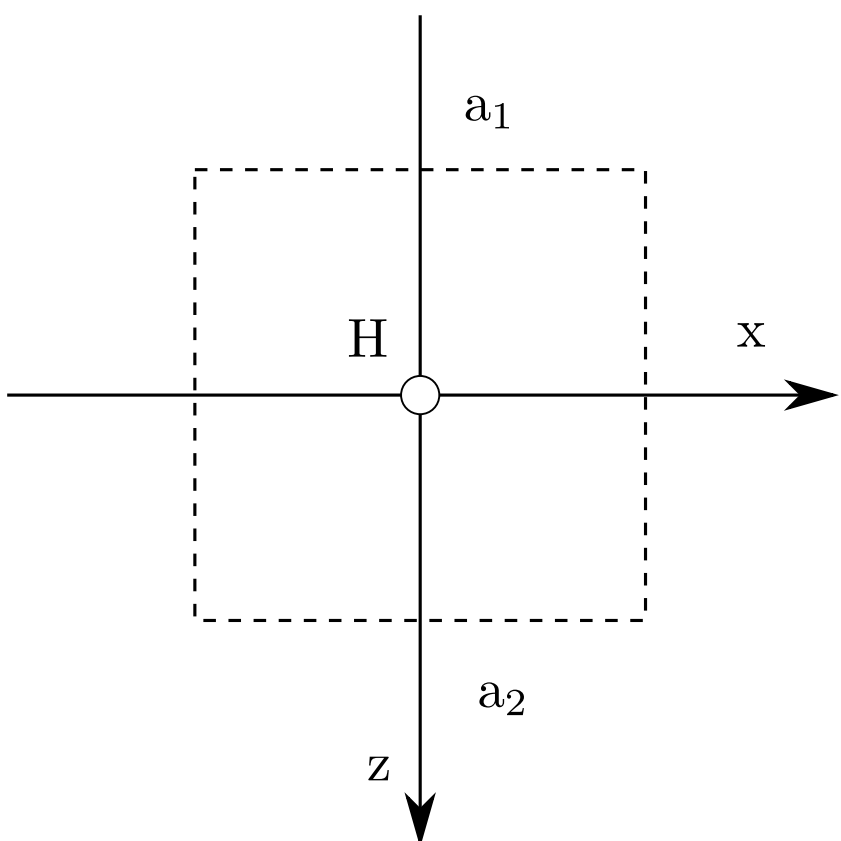

Fig. 1. Geometry of the Problem

The boundary conditions on the surface $z=0$ are (i) Mechanical condition

$$
\sigma_{z z}=\sigma_{z x}=0
$$

(ii) Concentration condition

$$
\frac{\partial C}{\partial z}=0
$$

(iii) Thermal condition

$$
\frac{\partial T}{\partial z}=0
$$

Introduce the harmonic functions for magnetothermoelastic diffusion material as

$$
\begin{array}{r}
\psi_{j}=A_{j}\left[\frac{1}{2}\left(z_{j}^{2}-x^{2}\right)\left(\log r_{j}-\frac{3}{2}\right)\right. \\
\left.-x z_{j} \tan ^{-1} \frac{x}{z_{j}}\right], \quad(j=1,2,3,4),
\end{array}
$$

where $A_{j}(\mathrm{j}=1,2,3,4)$ are arbitrary constants to be determined and

$$
r_{j}=\sqrt{x^{2}+z_{j}^{2}}, \quad(j=1,2,3,4)
$$

Substituting equation (42) in equations (33) and (39), we obtain the components of displacement, mass concentration, temperature change and stress components as follows

$$
u=-\sum_{j=1}^{4} A_{j}\left[x\left(\log r_{j}-1\right)+z_{j} \tan ^{-1} \frac{x}{z_{j}}\right],
$$

$$
\begin{gathered}
w=\sum_{j=1}^{4} s_{j} P_{1 j} A_{j}\left[z_{j}\left(\log r_{j}-1\right)-x \tan ^{-1} \frac{x}{z_{j}}\right] \\
C=\sum_{j=1}^{4} A_{j} P_{2 j} \log r_{j} \\
T=A_{4} P_{34} \log r_{4} \\
\sigma_{x x}=-\sum_{j=1}^{4} s_{j}^{2} w_{1 j} A_{j} \log r_{j} \\
\sigma_{z z}=\sum_{j=1}^{4} w_{1 j} A_{j} \log r_{j} \\
\sigma_{z x}=-\sum_{j=1}^{4} s_{j} w_{1 j} A_{j} \tan ^{-1} \frac{x}{z_{j}}
\end{gathered}
$$

Considering the continuity on plane $z=0$ for $w$ and $\sigma_{z x}$ yields

$$
\begin{aligned}
& \sum_{j=1}^{4} s_{j} P_{1 j} A_{j}=0, \\
& \sum_{j=1}^{4} s_{j} w_{1 j} A_{j}=0 .
\end{aligned}
$$

Substitution the values of $w_{1 j}$ from equation (40) in equation (46) gives

$$
\sum_{j=1}^{4} s_{j} A_{j}=0
$$

When the mechanical, concentration and thermal condition for a rectangle of $a_{1} \leq z \leq a_{2}\left(0<a_{1}<h<a_{2}\right)$ and $-b_{1} \leq x \leq b(b>0)$ are considered (Fig. 1), three equations can be obtained

$$
\begin{gathered}
\int_{-b}^{b}\left[\sigma_{z z}\left(x, a_{2}\right)-\sigma_{z z}\left(x, a_{1}\right)\right] \mathrm{d} x \\
+\int_{a_{1}}^{a_{2}}\left[\sigma_{z x}(b, z)-\sigma_{z x}(-b, z)\right] \mathrm{d} z=0, \\
\int_{-b}^{b}\left[\frac{\partial C}{\partial z}\left(x, a_{2}\right)-\frac{\partial C}{\partial z}\left(x, a_{2}\right)\right] \mathrm{d} x \\
+\int_{a_{1}}^{a_{2}}\left[\frac{\partial C}{\partial x}(b, z)-\frac{\partial C}{\partial x}(-b, z)\right] \mathrm{d} z=0, \\
-\varepsilon_{3} \int_{-b}^{b}\left[\frac{\partial T}{\partial z}\left(x, a_{2}\right)-\frac{\partial T}{\partial z}\left(x, a_{1}\right)\right] \mathrm{d} x \\
-\int_{a_{1}}^{a_{2}}\left[\frac{\partial T}{\partial x}(b, z)-\frac{\partial T}{\partial x}(-b, z)\right] \mathrm{d} z=H .
\end{gathered}
$$


Some useful integrals are given as below

$$
\begin{gathered}
\int \tan ^{-1}\left(\frac{x}{z_{j}}\right) \mathrm{d} z_{j}=x \log r_{j}+z_{j} \tan ^{-1}\left(\frac{x}{z_{j}}\right) \\
\int \log r_{j} \mathrm{~d} x=x\left(\log r_{j}-1\right)+z_{j} \tan ^{-1}\left(\frac{x}{z_{j}}\right), \\
\int \frac{\partial T}{\partial z} \mathrm{~d} x=s_{4} P_{34} A_{4} \int \frac{z_{4}}{r_{4}^{2}} \mathrm{~d} x= \\
=s_{4} P_{34} A_{4} \tan ^{-1} \frac{x}{z_{4}}, \\
\int \frac{\partial T}{\partial x} \mathrm{~d} z=P_{34} A_{4} \int \frac{x}{r_{4}^{2}} \mathrm{~d} z= \\
\int \frac{P_{34}}{s_{4}} A_{4} \tan ^{-1} \frac{x}{z_{4}}, \\
=\sum_{j=1}^{4} \mathrm{~d} x=\sum_{j=1}^{4} A_{j} s_{j}^{2} P_{2 j} \int \frac{z}{x^{2}+s_{j}^{2} z^{2}} \mathrm{~d} x= \\
=-\sum_{j=1} \frac{\tan ^{-1}}{s_{j}} \frac{x}{s_{j} z}, \\
P_{2 j} \tan ^{-1} \frac{x}{z_{j}}, \\
\mathrm{~d} z=\sum_{j=1}^{4} A_{j} P_{2 j} \int \frac{x}{x^{2}+s_{j}^{2} z^{2}} \mathrm{~d} z=
\end{gathered}
$$

It is noticed that the integrals $(49 \mathrm{~d}, \mathrm{~g})$ are not continuous at $z=0$, following expression should be used

$$
\begin{gathered}
\int_{a_{1}}^{a_{2}} \frac{\partial C}{\partial x} \mathrm{~d} z=\int_{a_{1}}^{0^{-}} \frac{\partial C}{\partial x} \mathrm{~d} z+\int_{0^{+}}^{a_{2}} \frac{\partial C}{\partial x} \mathrm{~d} z \\
\int_{a_{1}}^{a_{2}} \frac{\partial T}{\partial x} \mathrm{~d} z=\int_{a_{1}}^{0^{-}} \frac{\partial T}{\partial x} \mathrm{~d} z+\int_{0^{+}}^{a_{2}} \frac{\partial T}{\partial x} \mathrm{~d} z .
\end{gathered}
$$

Substituting equations (44 $\mathrm{f}, \mathrm{g})$ in equation (48a) and with the aid of the integrals ( $49 a, b)$, we obtain

$$
\sum_{j=1}^{4} w_{1 j} A_{j} I_{1}=0
$$

where

$$
\begin{gathered}
I_{1}=\left[\left(x\left(\log r_{j}-1\right)+s_{j} z \tan ^{-1} \frac{x}{s_{j} z}\right)_{z=a_{1}}^{z=a_{2}}\right]_{x=-b}^{x=b} \\
-\left[\left(x\left(\log r_{j}-1\right)+s_{j} z \tan ^{-1} \frac{x}{s_{j} z}\right)_{x=-b}^{x=b}\right]_{z=a_{1}}^{z=a_{2}}=0,
\end{gathered}
$$

i.e. Equations (48a) and (51) are satisfied automatically. Substituting the value of $C$ from equation (44c) in equation (49b) and using the integrals $(49 \mathrm{f}, \mathrm{g}$ ) and (50 a), we obtain

$$
\sum_{j=1}^{4} A_{j} P_{2 j} I_{2}=0
$$

where

$$
\begin{gathered}
I_{2}=\left[\left(s_{j}^{2} \tan ^{-1} \frac{x}{s_{j} z}\right)_{z=a_{1}}^{z=a_{2}}\right]_{x=-b}^{x=b} \\
-\left[\left(\tan ^{-1} \frac{x}{s_{j} z}\right)_{x=-b}^{x=b}\right]_{z=0_{1}}^{z=0^{-}} \\
-\left[\left(\tan ^{-1} \frac{x}{s_{j} z}\right)_{x=-b}^{x=b}\right]_{z=0^{+}}^{z=a_{2}}=\bar{r}_{j}
\end{gathered}
$$

Using equation (54) in equation (53), we obtain

$$
\sum_{j=1}^{4} \bar{r}_{j} A_{j} P_{2 j}=0 .
$$

Substituting equation (44d) in equation (48c) and using the integrals $(49 \mathrm{c}, \mathrm{d})$ and with the aid of equation (50b) and $s_{4}=\sqrt{\frac{K_{1}}{K_{3}}}$ on the resulting equation, we obtain

$$
A_{4} I_{3}=\frac{H}{P_{34} \sqrt{K_{3} / K_{1}}}
$$

where

$$
\begin{aligned}
& I_{2}=-\left[\left(\tan ^{-1}\left(\frac{x}{s_{4} z}\right)\right)_{z=a_{1}}^{z=a_{2}}\right]_{x=-b}^{x=b} \\
& +\left[\left(\tan ^{-1}\left(\frac{x}{s_{4} z}\right)\right)_{x=-b}^{x=b}\right]_{z=a_{1}}^{z=0^{-}} \\
& +\left[\left(\tan ^{-1}\left(\frac{x}{s_{4} z}\right)\right)_{x=-b}^{x=b}\right]_{z=0_{2}}^{z=a_{1}}=-2 \pi .
\end{aligned}
$$

$A_{4}$ can be determined from equation (56) and (57), as follows

$$
A_{4}=-\frac{H}{2 \pi P_{34} \sqrt{K_{3} / K_{1}}} .
$$

We have determined four constants $A_{j}(j=1,2,3,4)$ from four equations including equations (45), (47), (55) and (58) by the method of Cramer rule. 


\section{FUNDAMENTAL SOLUTION FOR A POINT HEAT SOURCE IN A SEMI-INFINITE ORTHOTROPIC MAGNETOTHERMOELASTIC DIFFUSION MATERIAL}

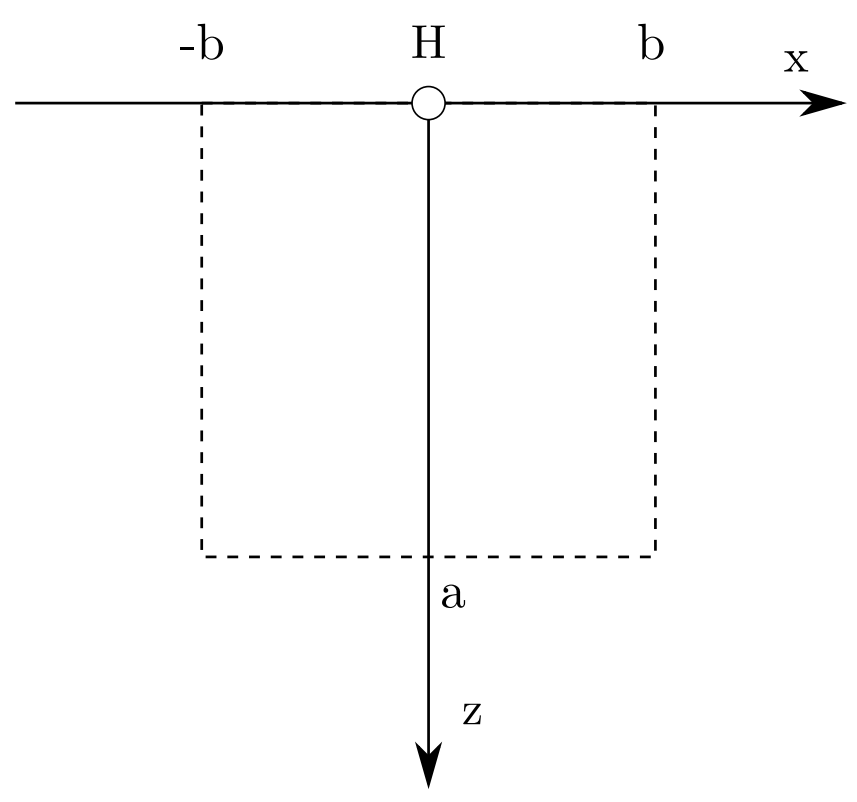

Fig. 2. Geometry of the Problem

As shown in Fig. 2, we consider a semi-infinite orthotropic magnetothermoelastic diffusion material $z \geq 0$. A point heat source $H$ is applied at the origin and the surface $z=0$ is free, impermeable boundary and thermally insulated. The complete geometry of the problem is shown in Fig. 2. The general solution given by equations (33) and (39) is derived in this section.

The boundary conditions on the surface $z=0$ are

$$
\sigma_{z z}=\sigma_{z x}=0, \quad \frac{\partial C}{\partial z}=0, \quad \frac{\partial T}{\partial z}=0
$$

When the mechanical, concentration and thermal condition for a rectangle of $0 \leq z \leq a$ and $-b \leq x \leq b(b>0)$ are considered (Fig. 2), three equations can be obtained

$$
\begin{gathered}
\int_{-b}^{b} \sigma_{z z}(x, a) \mathrm{d} x+\int_{0}^{a}\left[\sigma_{z x}(b, z)-\sigma_{z x}(-b, z)\right] \mathrm{d} z=0 \\
\int_{-b}^{b} \frac{\partial C}{\partial z}(x, a) \mathrm{d} x+\int_{0}^{a}\left[\frac{\partial C}{\partial x}(b, z)-\frac{\partial C}{\partial x}(-b, z)\right] \mathrm{d} z=0
\end{gathered}
$$

$$
\begin{aligned}
& -\varepsilon_{3} \int_{-b}^{b}\left[\frac{\partial T}{\partial z}(x, a)\right] \mathrm{d} x \\
& -\int_{a_{1}}^{a_{2}}\left[\frac{\partial T}{\partial x}(b, z)-\frac{\partial T}{\partial x}(-b, z)\right] \mathrm{d} z=H .
\end{aligned}
$$

The harmonic function given by equation (42) and expression for components of displacement, temperature distribution, mass concentration and stress components as given by equations (44a)-(44g) are the same, whereas the constants $A_{j}$ $(j=1,2,3,4)$ which are determined by boundary conditions (59) and equilibrium equations (50) are different. By substituting the values of $\sigma_{z z}, \sigma_{z x}, C$ and $T$ from equations (44 $\mathrm{c}, \mathrm{d}, \mathrm{f}, \mathrm{g}$ ) in equation (59), we obtain

$$
\begin{gathered}
\sum_{j=1}^{4} w_{1 j} A_{j}=0, \\
\sum_{j=1}^{4} s_{j} w_{1 j} A_{j}=0, \\
\sum_{j=1}^{4} \frac{z s_{j}^{2} p_{2 j}}{x^{2}+z_{j}^{2}} A_{j}=0 .
\end{gathered}
$$

Equation (61 c) shows that $\frac{\partial C}{\partial z}$ is satisfied automatically at the surface $z=0$ and similarly $\frac{\partial T}{\partial z}$ is also satisfied automatically at the surface $z=0$. Making use of the values of $\sigma_{z z}, \sigma_{z x}$ from equations (44 f, g) in equation (60 a) and using the integrals (49 a,b), we obtain

$$
\sum_{j=1}^{4} w_{1 j} A_{j} I_{4}=0
$$

where

$$
\begin{aligned}
& I_{3}=\left[x\left(\log \sqrt{x^{2}+s_{j}^{2} a^{2}}-1\right)+s_{j} a \tan ^{-1} \frac{x}{s_{j} a}\right]_{x=-b}^{x=b} \\
- & 2\left[z_{j} \tan ^{-1} \frac{b}{s_{j} z}+b \log \sqrt{b^{2}+s_{j}^{2} z^{2}}\right]_{z=0}^{z=a}= \\
= & 2 b(\log b-1) .
\end{aligned}
$$

By virtue of the equation (63), the equation (62) degenerates to equation (61a) i.e. equations (60a) and (62) are satisfied automatically.

$$
\begin{gathered}
A_{4} I_{5}=\frac{H}{P_{34} \sqrt{K_{3} / K_{1}}}, \\
I_{5}=-\left[\tan ^{-1}\left(\frac{x}{s_{4} a}\right)\right]_{x=-b}^{x=b} \\
+\left[\tan ^{-1}\left(\frac{b}{s_{4} z}\right)\right]_{z=0_{1}}^{z=a}=-\pi .
\end{gathered}
$$


$A_{4}$ can be determined from equation (64) and (65), as follows

$$
A_{4}=-\frac{H}{\pi P_{34} \sqrt{K_{3} / K_{1}}}
$$

Substituting the value of $C$ from equation (44c) in equation $(60 \mathrm{~b})$ and using the integrals ( $49 \mathrm{f}, \mathrm{g}$ ), we obtain

$$
\sum_{j=1}^{4} r_{j} A_{j} P_{2 j}=0
$$

where

$$
\begin{gathered}
r_{j}=\left[s_{j}^{2} \tan ^{-1}\left(\frac{x}{s_{j} a}\right)\right]_{x=-b}^{x=b} \\
-\left[\tan ^{-1}\left(\frac{b}{s_{j} z}\right)-\tan ^{-1}\left(\frac{-b}{s_{j} z}\right)\right]_{z=0}^{z=a} .
\end{gathered}
$$

We have determined four constants $A_{j}(j=1,2,3,4)$ from four equations, including equations (61 a), (61 b), (66) and (67) by the method of Cramer's rule.

\section{SPECIAL CASES}

(I) In the absence of magnetic effect, equations (44a)-(44f) reduces to

$$
\begin{gathered}
u=-\sum_{j=1}^{4} A_{j}\left[x\left(\log r_{j}-1\right)+z_{j} \tan ^{-1} \frac{x}{z_{j}}\right] \\
w=\sum_{j=1}^{4} s_{j} P_{1 j} A_{j}\left[z_{j}\left(\log r_{j}-1\right)-x \tan ^{-1} \frac{x}{z_{j}}\right], \\
C=\sum_{j=1}^{4} A_{j} P_{2 j} \log r_{j} \\
T=A_{4} P_{34} \log r_{4} \\
\sigma_{x x}=-\sum_{j=1}^{4} s_{j}^{2} w_{1 j} A_{j} \log r_{j} \\
\sigma_{z z}=\sum_{j=1}^{4} w_{1 j} A_{j} \log r_{j} \\
\sigma_{z x}=-\sum_{j=1}^{4} s_{j} w_{1 j} A_{j} \tan ^{-1} \frac{x}{z_{j}}
\end{gathered}
$$

In this case $s_{j}(j=1,2,3)$ are the roots of the (28) in the absence of magnetic effect. Which are similar to the results as those obtained by Kumar and Chawla [24].

(II) In the absence of magnetic and diffusion effect, equations (44a)-(44f) reduces to

$$
u=-\sum_{j=1}^{3} A_{j}\left[x\left(\log r_{j}-1\right)+z_{j} \tan ^{-1} \frac{x}{z_{j}}\right],
$$

$$
\begin{gathered}
w=\sum_{j=1}^{3} s_{j} P_{1 j} A_{j}\left[z_{j}\left(\log r_{j}-1\right)-x \tan ^{-1} \frac{x}{z_{j}}\right], \\
T=A_{4} P_{34} \log r_{4}, \\
\sigma_{x x}=-\sum_{j=1}^{3} s_{j}^{2} w_{1 j} A_{j} \log r_{j} \\
\sigma_{z z}=\sum_{j=1}^{3} w_{1 j} A_{j} \log r_{j} \\
\sigma_{z x}=-\sum_{j=1}^{3} s_{j} w_{1 j} A_{j} \tan ^{-1} \frac{x}{z_{j}} .
\end{gathered}
$$

In this case $s_{j}(j=1,2)$ are the roots of the (28) in the absence of magnetic and diffusion effect. The above results are similar as obtained by Hou et. al. [13].

\section{NUMERICAL RESULTS AND DISSCUSSION}

For the purpose of numerical computation, we take the following values of the relevant parameters as

$$
\begin{aligned}
& c_{11}=18.78 \times 10^{10} \mathrm{~kg} \mathrm{~m}^{-1} \mathrm{~s}^{-2}, \\
& c_{13}=8.0 \times 10^{10} \mathrm{~kg} \mathrm{~m}^{-1} \mathrm{~s}^{-2} \\
& c_{33}=10.2 \times 10^{10} \mathrm{~kg} \mathrm{~m}^{-1} \mathrm{~s}^{-2} \\
& c_{55}=10.06 \times 10^{10} \mathrm{~kg} \mathrm{~m}^{-1} \mathrm{~s}^{-2} \\
& T_{0}=0.293 \times 10^{3} \mathrm{~K} \\
& \alpha_{1}=1.96 \times 10^{-5} \mathrm{~K}^{-1} \\
& \alpha_{3}=1.4 \times 10^{-5} \mathrm{~K}^{-1} \\
& \alpha_{1 c}=1.1 \times 10^{-4} \mathrm{~m}^{3} \mathrm{~kg}^{-1} \\
& \alpha_{3 c}=0.43 \times 10^{-4} \mathrm{~m}^{3} \mathrm{~kg}^{-1} \\
& K_{1}=0.12 \times 10^{3} \mathrm{~W} \mathrm{~m}^{-1} \mathrm{~K}^{-1} \\
& K_{3}=0.33 \times 10^{3} \mathrm{~W} \mathrm{~m}^{-1} \mathrm{~K}^{-1} \\
& a=1.4 \times 10^{4} \mathrm{~m}^{2} \mathrm{~s}^{-2} \mathrm{~K}^{-1} \\
& b=9 \times 10^{5} \mathrm{~kg}^{-1} \mathrm{~m}^{5} \mathrm{~s}^{-2} \\
& \alpha_{1}^{*}=0.58 \times 10^{-8} \mathrm{~m}^{-3} \mathrm{~s} \mathrm{~kg}_{3} \\
& \alpha_{3}^{*}=0.52 \times 10^{-8} \mathrm{~m}^{-3} \mathrm{~s} \mathrm{~kg} \\
& H_{0}=0.38 \\
& \mu_{0}=1
\end{aligned}
$$




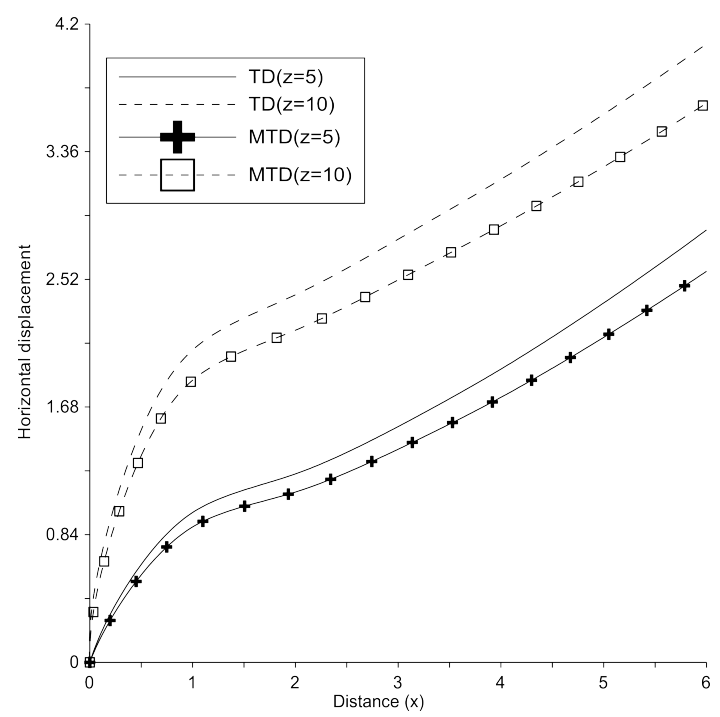

Fig. 3. Variation of horizontal displacement $(u)$ w.r.t. $x$ (for infinite plane)

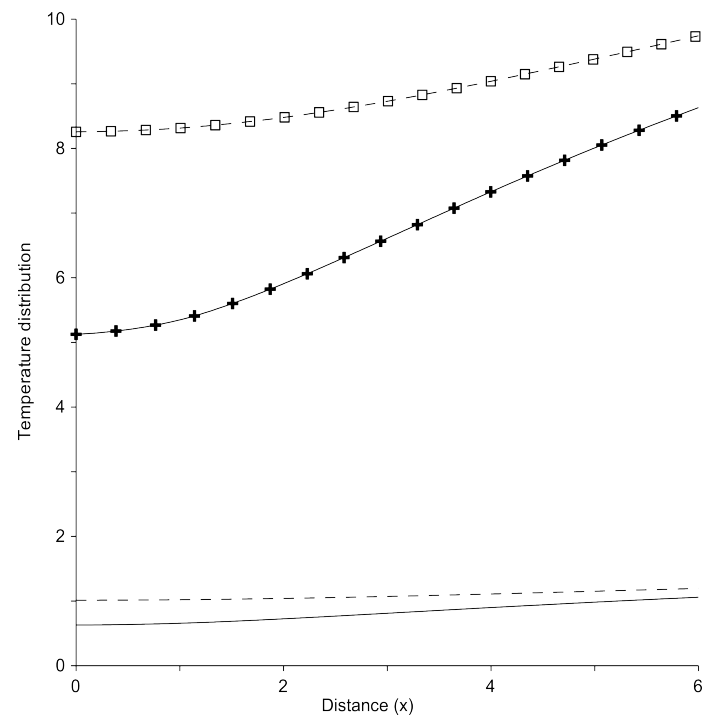

Fig. 5. Variation of temperature distribution $(T)$ w.r.t. $x$ (for infinite plane)

Figs. 3-6 depict the variation of components of displacement, temperature distribution and mass concentration for infinite magnetothermoelastic diffusion material and Figs. 7-10 exhibit the variation of components of displacement, temperature distribution and mass concentration for semi-infinite magnetothermoelastic diffusion material. The solid lines and dotted lines correspond to thermoelastic diffusion material (TD) and center symbol on these lines correspond to magnetothermoelastic diffusion material (MTD).

Fig. 3 depicts the variation of horizontal displacement $u$ with $x$. It is noticed that the values of $u$ increase for smaller values of $x$, but for higher values of $x$, the values of $u$ increase monotonically. It is evident that the values of $u$ in

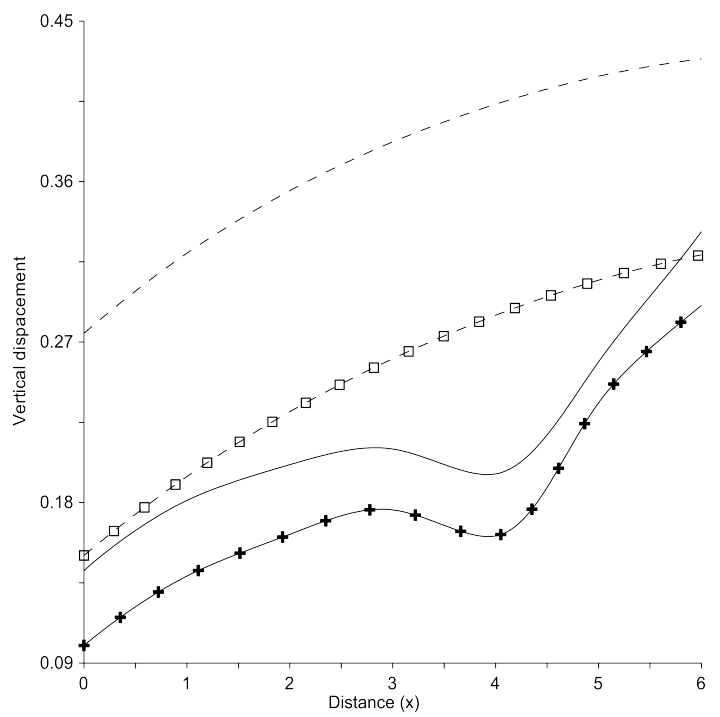

Fig. 4. Variation of vertical dispacement $(w)$ w.r.t. $x$ (for infinite plane)

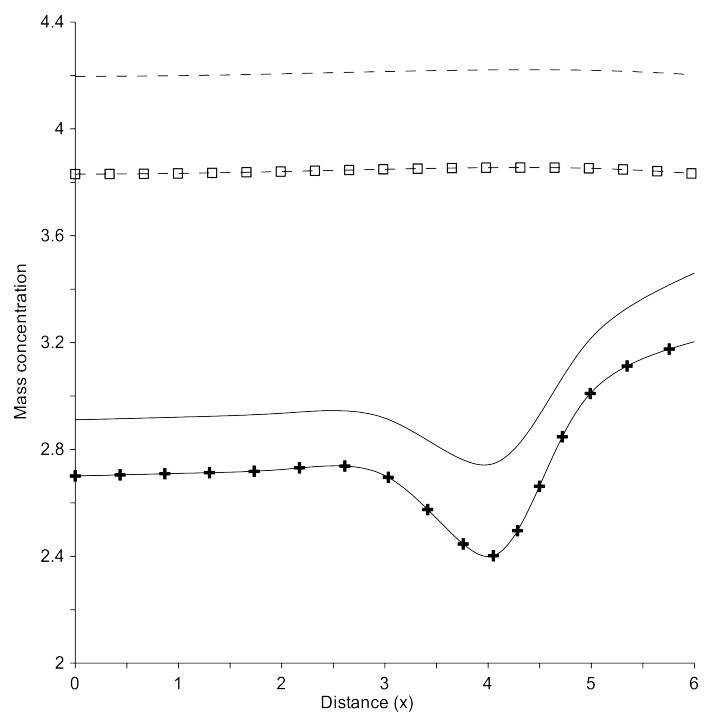

Fig. 6. Variation of mass concentration w.r.t. $x$ (for infinite plane)

case of TD $(z=5)$ remain more (in comparison with MTD $(z=5))$ and similar behavior is observed for the cases of $\operatorname{TD}(z=5)$ and MTD $(z=10)$. Fig. 4 depicts the variation of vertical displacement $(w)$ with $x$. It indicates that the values of $w$ increase for the cases of TD and MTD $(z=10)$, although for the cases of TD, MTD $(z=5)$, the values of $w$ remain oscillatory for smaller values of $x$, but for higher values of $x$, the values of $w$ increase monotonically. It is observed that the values of $w$ in case of $\operatorname{TD}(z=10)$ remain more in comparison with others.

Fig. 5 exhibits the variation of temperature distribution (T) with $x$. It is noticed that the values of $T$ for both cases TD and MTD increase for all values of $x$. It is evident that 
the values of $T$ in case of MTD remain more in comparison with TD. Fig. 6 depicts the variation of mass concentration $(C)$ with $x$, and it indicates that for the cases of TD and MTD $(z=10)$, the values of $C$ decrease slightly, although for the

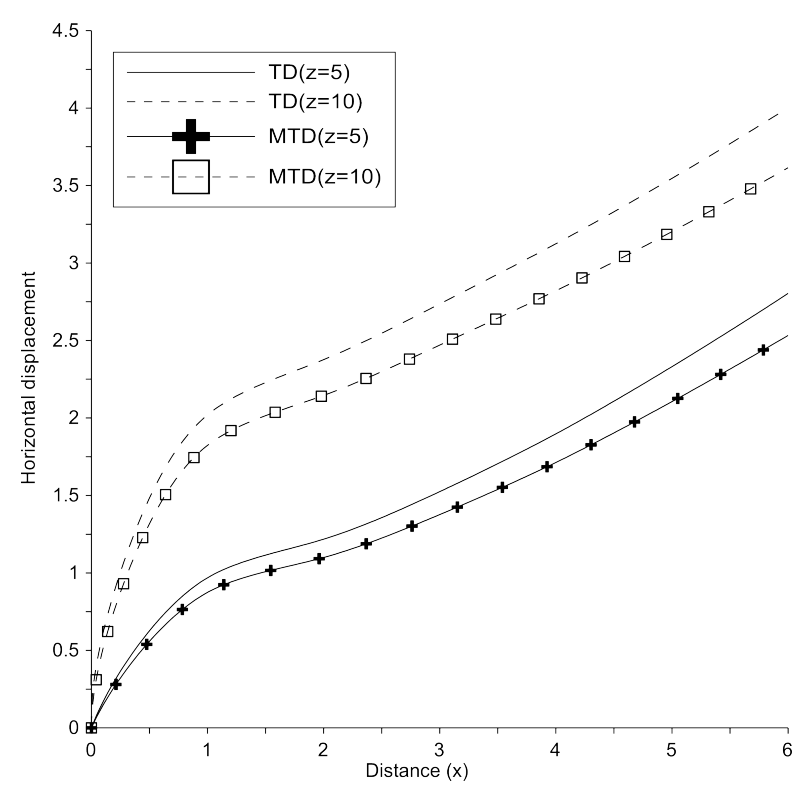

Fig. 7. Variation of horizontal displacement w.r.t. $x$ (for semiinfinite plane)

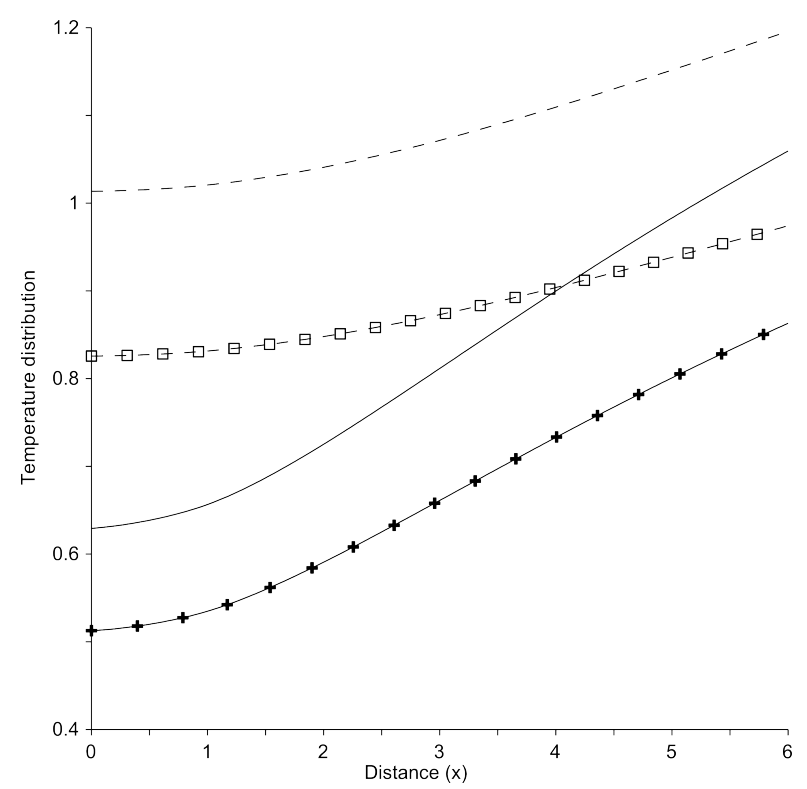

Fig. 9. Variation of temperature distribution $(T)$ w.r.t. $x$ (for semi-infinite plane)

Fig. 7 exhibits the variation of horizontal displacement (u) with $x$. It is evident that the behavior and variation of $u$ for the semi-infinite magnetothermoelastic diffusion material is similar as for the infinite magnetothermoelastic dif- case of TD and MTD $(z=5)$ the values of $C$ oscillates for smaller values of $x$, but for higher values of $x$, the values of $C$ increase. It is evident that the values of $C$ for $\operatorname{TD}(z=10)$ remain more in comparison with others.

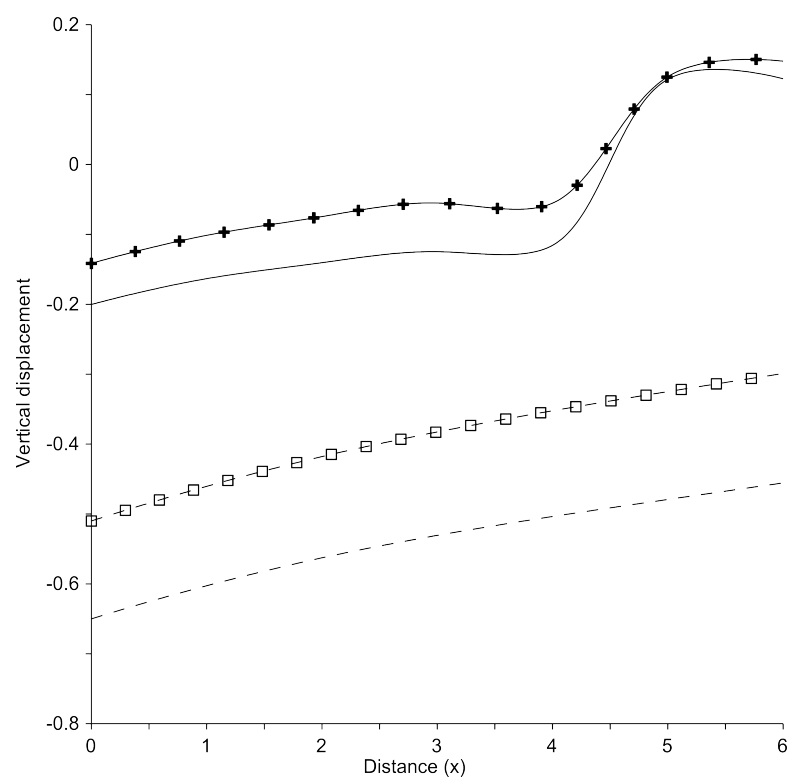

Fig. 8. Varition of Vertical displacement $(w)$ w.r.t. $x$ (for semiinfinite plane)

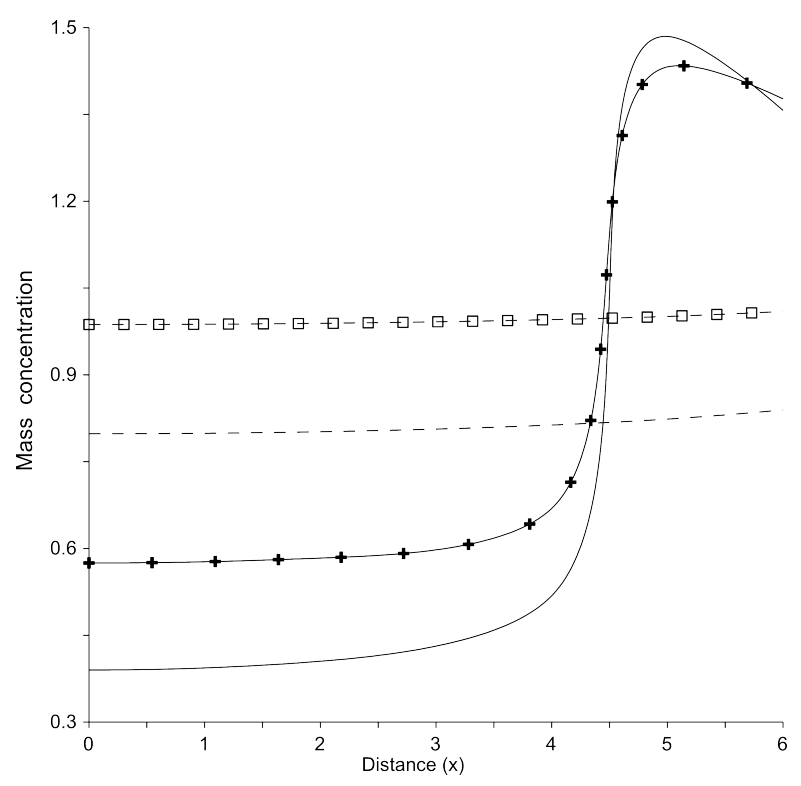

Fig. 10. Variation of mass concentration $(C)$ w.r.t. $x$ (for semiinfinite plane)

fusion material, although the magnitude values of $u$ in case of semi-infinite magnetothermoelastic diffusion material are different from the infinite magnetothermoelastic diffusion material. Fig. 8 depicts the variation of vertical displacement 
( $w$ ) with $x$, and it shows that the values of $w$, for the cases of cases $\operatorname{TD}(z=5)$, MTD $(z=5)$ increase for all values of $x$, whereas for the cases of TD and MTD $(z=10)$, the values of $w$ increase for smaller values of $x$, but for higher values of $x$, the values of $w$ remain oscillatory. It is evident that the values of $w$ in case of $\operatorname{MTD}(z=5)$ and $\operatorname{MTD}(z=10)$ remain more (in comparison with TD $(z=5)$ and TD $(z=10)$ ).

Fig. 9 depicts the variation of temperature distribution $(T)$ with $x$, and it indicates that, for both cases TD and MTD, the values of $T$ increase. It is noticed that the values of $T$ in case of TD $(z=10)$ remain more in comparison with $\operatorname{TD}(z=5)$ and MTD $(z=5, z=10)$. Fig. 10 depicts the variation of mass concentration $(C)$ with $x$, and it shows that the values of $C$ slightly increase for the case of TD, MTD $(z=10)$ whereas for the case of TD, MTD $(z=5)$, the values of $C$ remain oscillatory.

\section{CONCLUDING REMARKS}

The fundamental solution for two-dimensional in orthotropic magnetothermoelastic diffusion material has been derived. With this objective, the two-dimensional general solution in magnetothermoelastic diffusion material is derived firstly for the case of distinct roots. By virtue of the two-dimensional general solution of orthotropic magnetothermoelastic diffusion material, the fundamental solution for a steady point heat source acting in an infinite plane and on the surface of a semi-infinite plane are obtained by four newly introduced harmonic functions $\psi_{j}(j=1,2,3,4)$. The general expression for components of displacement, stress, mass concentration and temperature change are expressed in terms of elementary functions. Since all the components are expressed in terms of elementary functions, it is convenient to use them. From the present investigation, some special cases of interest are also deduced. The components of displacement, mass concentration and temperature distribution are computed numerically and depicted graphically to depict the magnetic effect.

From the numerical results we conclude that, due to the magnetic effect, the values of horizontal displacement $(u)$, vertical displacement $(w)$ and mass concentration $(C)$ become smaller, although for the values to $T$ become higher due to the magnetic effect. In case of the semi-infinite plane it is noticed that, due to themagnetic effect, the values of horizontal displacement $(u)$ and temperature change $(T)$ become smaller, whereas the values of mass concentration $(C)$ become higher due to the magnetic effect. Appreciable magnetic effect is observed on components of displacement, temperature distribution and mass concentration for the infinite magnetothermoelastic diffusion material and semi-infinite magnetothermoelastic diffusion material.

\section{References}

[1] H.J.Ding, B.Chen, J. Liang, General Solutions for Coupled Equations in Piezoelectric Media, International Journal of Solids and Structures 33, 2283-2298 (1996).

[2] M.L.Dunn, H.A.Wienecke, Half Space Green's Functions for Transversely Isotropic Piezoelectric Solids, Journal of Applied Mechanics 66, 675-679 (1999).

[3] E. Pan, F. Tanon, Three Dimensional Green's Functions in Anisotropic Piezoelectric Solids, International Journal of Solids and Structures 37, 943-958 (2000).

[4] H.J.Ding, F.L.Guo, and P.F.Hou, A general Solution for Piezothermoelasticity of Transversely Isotropic Piezoelectric Materials and its Applications, International Journal of Engineering Science 38, 1415-1440 (2000).

[5] B. Sharma, Thermal Stresses in Transversely Isotropic SemiInfinite Elastic Solids, ASME Journal of Applied Mechanics 23, 86-88 (1958).

[6] W.Q. Chen, H.J. Ding, D.S. Ling, Thermoelastic field of Transversely Isotropic Elastic Medium Containing a PennyShaped Crack: Exact Fundamental Solution, International Journalof Solids and Structures 41, 69-83 (2004).

[7] P.F. Hou, A.Y.T. Leung, C.P. Chen, Green's Functions for Semi-Infinite Transversely Isotropic Thermoelastic Materials, ZAMM Z. Angew. Math. Mech. 1, 33-41 (2008).

[8] P.F. Hou, L. Wang, T. Yi, 2D Green's Functions for SemiInfinite Orthotropic Thermoelastic Plane, Applied Mathematical Modeling 33, 1674-1682 (2009).

[9] P.F. Hou, H. Sha, C.P. Chen, 2D General Solution and Fundamental Solution for Orthotropic Thermoelastic Materials, Engineering.Analysis with Boundary Elements 45, 392-408 (2008).

[10] S. Kaloski, W. Nowacki, Wave Propagation of ThermoMagneto-Microelasticity, Bulletin of the Polish Academy of Sciences Technical Sciences 18 155-158 (1970).

[11] M.I.A.Othman, Y. Song, Reflection of MagnetoThermoelastic Waves with Two-Relaxation Times and Temperature Dependent Elastic Moduli, Applied Mathematical Modeling 32,483-500 (2008).

[12] P.F. Hou, T. Yi, L.Wang, 2D General Solution and Fundamental Solution for Orthotropic Electro-Magneto-Thermoelastic Materials, Journal of Thermal Stresses 31, 807-822 (2008).

[13] W. Nowacki, Dynamical Problem of Thermodiffusion in Solid - I, Bulletin ofpolish Academy of Sciences Series, Science and Technology 22, 55-64 (1974).

[14] W. Nowacki, Dynamical Problem of Thermodiffusion in Solid - II, Bulletin of Polish Academy of Sciences Series, Science and Technology 22, 129-135 (1974).

[15] W. Nowacki, Dynamical Problem of Thermodiffusion in Solid - III, Bulletin of Polish Academy of Sciences Series, Science and Technology 22, 275-276 (1974).

[16] W. Nowacki, Dynamical Problems of Thermodiffusion in Solids, Proc. Vib. Prob. 15, 105-128 (1974).

[17] H.H. Sherief, H. Saleh, A Half Space Problem in the Theory of Generalized Thermoelastic Diffusion, International Journal of Solids and Structures 42, 4484-4493 (2005).

[18] R. Kumar, T. Kansal, Propagation of Lamb waves in transversely isotropic Thermoelastic diffusive plate, Int. J. Solid Struct. 45 (2008), pp. 5890-5913.

[19] R. Kumar, V. Chawla, A Study of Plane Wave Propagation in Anisotropic Three-Phase-Lag and Two-Phase-Lag Model, International Communication in Heat and Mass Transfer 38, 1262-1268 (2011).

[20] R. Kumar, V. Chawla, A Study of Fundamental Solution in Orthotropic Thermodiffusive Elastic Media, International Communication in Heat and Mass Transfer 38, 456-462 (2011). 
[21] R. Kumar, V. Chawla, Green's Functions in Orthotropic ThermoelasticDiffusion Media, Engineering Analysis with Boundary Elements 36, 1272-1277 (2012).

[22] M.A. Ezzat, State approach to generalized magnetothermoelastic with two-relaxation times in a medium of perfect conductivity, International Journal of Engineering Science 35, 741-752 (1997).

[23] A.C. Eringen, Foundations and Solids, Microcontinuum Field Theories, Springer-Verlag, New York 1999.

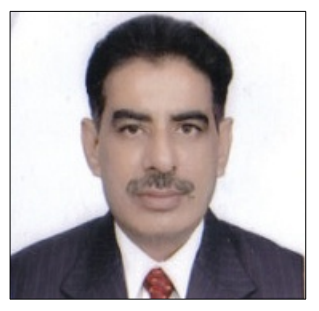

Rajneesh Kumar, born on 8th June 1958, received his MSc (1980) from Guru Nanak Dev University, Amritsar (Punjab, India), M. Phil. (1982) from Kurukshetra University Kurukshetra (Haryana, India) and PhD (1986) in Applied Mathematics from Guru Nanak Dev University, Amritsar (Punjab, India). He has supervised 52 M. Phil. students, 15 students awarded a $\mathrm{PhD}$ degree and 8 students are doing $\mathrm{PhD}$ under his supervision. He boasts 330 publications in journals of international repute. His area of research work is Continuum Mechanics (micropolar elasticity, thermoelasticity, poroelasticity, magnetoelasticity, micropolar porous couple stress theory, viscoelasticity, mechanics of fluid.

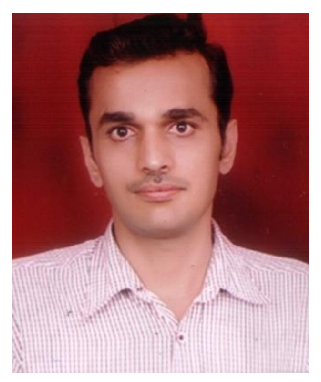

Vijay Chawla, born on 7th July 1984, received the MSc degree (2007) from Kurukshetra University, Kurukshetra (Haryana, India) and PhD (April 2013) in Applied Mathematics from Kurukshetra University, Kurukshetra (Haryana, India). Presently working as Assistant Professor in Swami Devi Dyal Institute of Engineering and Technology, Barwala (Panchkula, Haryana). 